\title{
A counterexample to a conjecture of Selmer
}

\author{
Tom Fisher
}

6 November 2002

\begin{abstract}
We present a counterexample to a conjecture cited by Cassels [CaI] and attributed to Selmer. The issues raised have been given new significance by the recent work of Heath-Brown [HB] and SwinnertonDyer $[\mathrm{SwD}]$ on the arithmetic of diagonal cubic surfaces.
\end{abstract}

\section{Introduction}

Let $E$ be an elliptic curve over a number field $k$, with complex multiplication by $\mathbf{Z}[\omega]$ where $\omega$ is a primitive cube root of unity. Let $K=k(\omega)$, so that $[K: k]=1$ or 2 according as $\omega \in k$ or $\omega \notin k$. In his work on cubic surfaces, Heath-Brown [HB] makes implicit use of

Theorem 1.1 If $[K: k]=2$ and the Tate-Shafarevich group $\amalg(E / k)$ is finite, then the order of $\amalg(E / K)[\sqrt{-3}]$ is a perfect square.

We explain how this result follows from the work of Cassels [CaIV], and give an example to show that the condition $[K: k]=2$ is necessary.

For the application to cubic surfaces, we only need a special case of the theorem, namely that $\amalg(E / K)[\sqrt{-3}]$ cannot have order 3 . This result, still conditional on the finiteness of the Tate-Shafarevich group, has already appeared in $[\mathrm{BF}]$ and $[\mathrm{SwD}]$. In fact Swinnerton-Dyer $[\mathrm{SwD}]$ vastly generalises Heath-Brown's results. In the case $[K: k]=2$ he proves the Hasse principle for diagonal cubic 3-folds over $k$, conditional only on the finiteness of the Tate-Shafarevich group for elliptic curves over $k$. The condition $[K: k]=2$ is unnatural, and conjecturally should not appear. However, the counterexample presented in this article suggests that, if we are to follow the methods of Heath-Brown and Swinnerton-Dyer, then this condition on $k$ is unavoidable. 
In $\S 2$ we recall how it is possible to pass between the fields $k$ and $K$. Then in $\S 3$ we give a modern treatment of the descent by 3 -isogeny studied by Selmer [S1] and Cassels [CaI]. In $\S \S 4,5$ we recall how the conjectures of Selmer may be deduced from properties of the Cassels-Tate pairing. This culminates in a proof of Theorem 1.1. Finally in $\S 6$ we present our new example.

\section{Decomposition into Galois eigenspaces}

Let $E$ be an elliptic curve over $k$ with complex multiplication by $\mathbf{Z}[\omega]$. The isogeny $[\sqrt{-3}]: E \rightarrow E$ is defined over $K=k(\omega)$. But the kernel $E[\sqrt{-3}]$ is defined over $k$. It follows that there is a 3-isogeny $\phi: E \rightarrow \widetilde{E}$ defined over $k$ with $E[\sqrt{-3}]=E[\phi]$. Here $\widetilde{E}$ is a second elliptic curve defined over $k$, which we immediately recognise as the -3 -twist of $E$. The dual isogeny $\widehat{\phi}: \widetilde{E} \rightarrow E$ satisfies $\phi \circ \widehat{\phi}=[3]$ and $\widehat{\phi} \circ \phi=[3]$. Our notation for the Selmer groups and Tate-Shafarevich groups follows [Sil, Chapter X].

Lemma 2.1 If $[K: k]=2$ then the exact sequence

$$
0 \longrightarrow E(K) / \sqrt{-3} E(K) \longrightarrow S^{(\sqrt{-3})}(E / K) \longrightarrow \amalg(E / K)[\sqrt{-3}] \longrightarrow 0
$$

is the direct sum of the exact sequences

$$
0 \longrightarrow \widetilde{E}(k) / \phi E(k) \longrightarrow S^{(\phi)}(E / k) \longrightarrow \amalg(E / k)[\phi] \longrightarrow 0
$$

and

$$
0 \longrightarrow E(k) / \widehat{\phi} \widetilde{E}(k) \longrightarrow S^{(\widehat{\phi})}(\widetilde{E} / k) \longrightarrow \amalg(\widetilde{E} / k)[\widehat{\phi}] \longrightarrow 0 .
$$

Proof. Since arguments of this type have already appeared in $[\mathrm{BF}],[\mathrm{N}]$, $[\mathrm{SwD}]$ and presumably countless other places in the literature, we will not dwell on the proof. Suffice to say that we decompose (1) into eigenspaces for the action of $\operatorname{Gal}(K / k)$, and then use the inflation-restriction exact sequence to identify these eigenspaces as (2) and (3). The observation that $[K: k]=2$ is prime to $\operatorname{deg} \phi=3$ is crucial throughout the proof.

Remark 2.2 Each term of the exact sequence (1) is a $\mathbf{Z} / 3 \mathbf{Z}$-vector space with an action of $\operatorname{Gal}(K / k)$. So each term is a direct sum of the Galois modules $\mathbf{Z} / 3 \mathbf{Z}$ and $\mu_{3}$. If we replace $E$ by $\widetilde{E}$ in (1) then we obtain the same exact sequence of abelian groups, but as Galois modules the summands $\mathbf{Z} / 3 \mathbf{Z}$ and $\mu_{3}$ are interchanged. 


\section{Computation of Selmer groups}

Let $k$ be a number field. Let $T\left[a_{0}, a_{1}, a_{2}\right]$ be the diagonal plane cubic

$$
a_{0} x_{0}^{3}+a_{1} x_{1}^{3}+a_{2} x_{2}^{3}=0
$$

where $a_{0}, a_{1}, a_{2} \in k^{*} / k^{* 3}$. Let $E_{A}$ be the elliptic curve $T[A, 1,1]$ with identity element $0=(0: 1:-1)$. It is well known $[\mathrm{St}]$ that $E_{A}$ has Weierstrass equation $y^{2}=x^{3}-432 A^{2}$. An alternative proof of the following lemma may be found in [CaL, $\S 18]$.

Lemma 3.1 The diagonal plane cubic $T\left[a_{0}, a_{1}, a_{2}\right]$ is a smooth curve of genus 1 with Jacobian $E_{A}$ where $A=a_{0} a_{1} a_{2}$.

Proof. There is an isomorphism $T\left[a_{0}, a_{1}, a_{2}\right] \simeq E_{A}$ defined over $k(\sqrt[3]{\alpha})$ where $\alpha=a_{1} a_{2}^{2}$. It is given by

$$
\psi:\left(x_{0}: x_{1}: x_{2}\right) \mapsto\left(a_{2} x_{0}: \alpha^{2 / 3} x_{1}: \alpha^{1 / 3} a_{2} x_{2}\right) .
$$

The cocycle $\sigma(\psi) \psi^{-1}$ takes values in the subgroup $\mu_{3} \subset \operatorname{Aut}\left(E_{A}\right)$ generated by $x_{i} \mapsto \omega^{i} x_{i}$. But since $\mu_{3}$ acts on $E_{A}$ without fixed points, this action belongs to the translation subgroup of $\operatorname{Aut}\left(E_{A}\right)$. It follows that $T\left[a_{0}, a_{1}, a_{2}\right]$ is a torsor under $E_{A}$ and that $E_{A}$ is the Jacobian of $T\left[a_{0}, a_{1}, a_{2}\right]$.

Temporarily working over $K=k(\omega)$ we note that $E_{A}$ has complex multiplication by $\mathbf{Z}[\omega]$ where $\omega:\left(x_{0}: x_{1}: x_{2}\right) \mapsto\left(\omega x_{0}: x_{1}: x_{2}\right)$ and that $E_{A}[1-\omega]=E_{A}[\sqrt{-3}]$ is generated by $\left(0: \omega:-\omega^{2}\right)$. So as in $\S 2$ there is a map $\phi$ which gives an exact sequence of Galois modules

$$
0 \longrightarrow \mu_{3} \longrightarrow E_{A} \stackrel{\phi}{\longrightarrow} \widetilde{E}_{A} \longrightarrow 0
$$

where $\widetilde{E}_{A}$ is the -3 -twist of $E_{A}$. Taking Galois cohomology we obtain an exact sequence

$$
0 \longrightarrow \widetilde{E}_{A}(k) / \phi E_{A}(k) \stackrel{\delta}{\longrightarrow} k^{*} / k^{* 3} \longrightarrow H^{1}\left(k, E_{A}\right)[\phi] \longrightarrow 0 .
$$

The group $H^{1}\left(k, E_{A}\right)$ parametrises the torsors under $E_{A}$. We write $C_{A, \alpha}$ for the torsor under $E_{A}$ described by $\alpha \in k^{*} / k^{* 3}$. The proof of Lemma 3.1 shows that

$$
T\left[a_{0}, a_{1}, a_{2}\right] \simeq C_{A, \alpha} \quad \text { for } A=\prod a_{\nu} \text { and } \alpha=\prod a_{\nu}^{\nu}
$$


where the products are over $\nu \in \mathbf{Z} / 3 \mathbf{Z}$. Since $T\left[a_{0}, a_{1}, a_{2}\right] \simeq T\left[a_{1}, a_{2}, a_{0}\right]$ it is clear that $A \in \operatorname{im} \delta$. If $\widetilde{E}_{A}$ has Weierstrass equation $Y^{2} Z=-4 A X^{3}+Z^{3}$ then the 3 -covering map $T\left[a_{0}, a_{1}, a_{2}\right] \rightarrow \widetilde{E}_{A}$ is given by

$$
\left(x_{0}: x_{1}: x_{2}\right) \mapsto\left(x_{0} x_{1} x_{2}: a_{1} x_{1}^{3}-a_{2} x_{2}^{3}: a_{0} x_{0}^{3}\right) .
$$

The Selmer group attached to $\phi$ is

$$
S^{(\phi)}\left(E_{A} / k\right)=\left\{\alpha \in k^{*} / k^{* 3} \mid C_{A, \alpha}\left(k_{\mathfrak{p}}\right) \neq \emptyset \text { for all primes } \mathfrak{p}\right\} .
$$

Since $\operatorname{deg} \phi=3$ is odd we have ignored the infinite places. We write $\delta_{\mathfrak{p}}$ for the local connecting map obtained when we apply (5) to the local field $k_{\mathrm{p}}$. Then the condition $C_{A, \alpha}\left(k_{\mathfrak{p}}\right) \neq \emptyset$ may also be written $\alpha \in \operatorname{im} \delta_{\mathfrak{p}}$. Using (6) to give equations for $C_{A, \alpha}$ it is easy to prove

Lemma 3.2 Let $k$ be a number field, and let $\mathfrak{p}$ be a prime not dividing 3. Let $\mathfrak{o}_{\mathfrak{p}}$ denote the ring of integers of $k_{\mathfrak{p}}$. Then

$$
\operatorname{im} \delta_{\mathfrak{p}}=\left\{\begin{array}{ccc}
\mathfrak{o}_{\mathfrak{p}}^{*} / \mathfrak{o}_{\mathfrak{p}}^{* 3} & \text { if } \operatorname{ord}_{\mathfrak{p}}(A) \equiv 0 & (\bmod 3) \\
\langle A\rangle & \text { if } \operatorname{ord}_{\mathfrak{p}}(A) \not \equiv 0 & (\bmod 3)
\end{array}\right.
$$

If $\mathfrak{p}$ divides 3 the situation is more complicated, although we still have

$$
\operatorname{im} \delta_{\mathfrak{p}} \subset \mathfrak{o}_{\mathfrak{p}}^{*} / \mathfrak{o}_{\mathfrak{p}}^{* 3} \quad \text { if } \operatorname{ord}_{\mathfrak{p}}(A) \equiv 0 \quad(\bmod 3) .
$$

If $\omega \in k_{\mathfrak{p}}$ then Tate local duality tells us that $\operatorname{im} \delta_{\mathfrak{p}}$ is a maximal isotropic subspace with respect to the Hilbert norm residue symbol

$$
k_{\mathfrak{p}}^{*} / k_{\mathfrak{p}}^{* 3} \times k_{\mathfrak{p}}^{*} / k_{\mathfrak{p}}^{* 3} \rightarrow \mu_{3} .
$$

The next lemma treats the case $k=\mathbf{Q}(\omega)$. This field has ring of integers $\mathbf{Z}[\omega]$ and class number 1 . The unique prime above 3 is $\pi=\omega-\omega^{2}$.

Lemma 3.3 Let $A \in \mathbf{Z}[\omega]$ be non-zero and cube-free. Then

$$
\operatorname{im} \delta_{\pi}=\left\{\begin{array}{cl}
\langle A,(1-A) /(1+A)\rangle & \text { if } \operatorname{ord}_{\pi}(A) \neq 0 \\
\left\langle A, 1-\pi^{3}\right\rangle & \text { if } \operatorname{ord}_{\pi}(A)=0 \text { and } A^{2} \not \equiv \pm 1\left(\pi^{3}\right) \\
\left\langle\omega(1+3 a), 1-\pi^{3}\right\rangle & \text { if } A= \pm\left(1+a \pi^{3}\right) \text { for some } a \in \mathbf{Z}[\omega] .
\end{array}\right.
$$


Proof. We recall [CF, Exercise 2.13] that $k_{\pi}^{*} / k_{\pi}^{* 3}$ has basis $\pi, \omega, 1-\pi^{2}, 1-\pi^{3}$ and that these elements define a filtration compatible with the pairing (8). By Tate local duality it follows that $\operatorname{im} \delta_{\pi}$ has order 9 . So to prove the lemma it suffices to prove the inclusions $\supset$. As always $A \in \operatorname{im} \delta_{\pi}$, whereas (7) and Tate local duality tell us that $1-\pi^{3} \in \operatorname{im} \delta_{\pi}$. There is at most one more element to find.

(i) $\operatorname{Suppose}_{\operatorname{ord}}(A) \neq 0$. If $\alpha$ satisfies $\alpha-\alpha^{-1}=A$ then $T\left[A, \alpha, \alpha^{-1}\right]$ is soluble. Splitting into the cases $\operatorname{ord}_{\pi}(A)=1$ or 2 we find

$$
4 A /\left(1-A^{2}\right) \equiv A \quad\left(\bmod \pi^{4}\right) .
$$

So $\alpha=(1-A) /(1+A)$ provides a solution $\bmod \pi^{4}$.

(ii) Suppose $A=1+a \pi^{3}$ for some $a \in \mathbf{Z}[\omega]$. If $\alpha$ satisfies $A+\alpha+\alpha^{-1}=0$ then $T\left[A, \alpha, \alpha^{-1}\right]$ is soluble. In view of the identity

$$
\left(1+\pi^{3} a\right)+\omega(1+3 a)+\omega^{2}(1-3 a)=0
$$

we see that $\alpha=\omega(1+3 a)$ provides a solution $\bmod \pi^{4}$.

\section{Selmer's conjectures}

In this section we take $k=\mathbf{Q}$, so that $K=\mathbf{Q}(\omega)$. We consider the elliptic curves $E_{A}$ and $\widetilde{E}_{A}$ over $\mathbf{Q}$ where $A \geq 2$ is a cube-free integer.

Lemma 4.1 If $A \geq 3$ then the torsion subgroups are

$$
E_{A}(\mathbf{Q})_{\text {tors }}=0 \quad \text { and } \quad \widetilde{E}_{A}(\mathbf{Q})_{\text {tors }} \simeq \mathbf{Z} / 3 \mathbf{Z} .
$$

Proof. See [St, §6] or [K, Chapter 1, Problem 7].

Lemma 2.1 gives a decomposition into $\mathrm{Gal}(K / \mathbf{Q})$-eigenspaces

$$
S^{(\sqrt{-3})}\left(E_{A} / K\right) \simeq S^{(\phi)}\left(E_{A} / \mathbf{Q}\right) \oplus S^{(\widehat{\phi})}\left(\widetilde{E}_{A} / \mathbf{Q}\right) .
$$

The following examples were found by Selmer [S1], [S2].

Example 4.2 Let $A=60$. Lemmas 3.2 and 3.3 tell us that

$$
S^{(\sqrt{-3})}\left(E_{60} / K\right) \simeq\langle 2,3,5\rangle \subset K^{*} / K^{* 3} .
$$


Then (9) gives $S^{(\phi)}\left(E_{60} / \mathbf{Q}\right) \simeq(\mathbf{Z} / 3 \mathbf{Z})^{3}$ and $S^{(\widehat{\phi})}\left(\widetilde{E}_{60} / \mathbf{Q}\right)=0$. But a 2-descent [CaL, $§ 15],[\mathrm{Cr}]$ shows that $E_{60}(\mathbf{Q})$ has rank 0 . We deduce

$$
\amalg\left(E_{60} / \mathbf{Q}\right)[3] \simeq(\mathbf{Z} / 3 \mathbf{Z})^{2} .
$$

Example 4.3 Let $A=473$. Lemmas 3.2 and 3.3 tell us that

$$
S^{(\sqrt{-3})}\left(E_{473} / K\right) \simeq\left\langle 11,1-6 \omega, 1-6 \omega^{2}\right\rangle \subset K^{*} / K^{* 3} .
$$

Then (9) gives $S^{(\phi)}\left(E_{473} / \mathbf{Q}\right) \simeq(\mathbf{Z} / 3 \mathbf{Z})^{2}$ and $S^{(\widehat{\phi})}\left(\widetilde{E}_{473} / \mathbf{Q}\right) \simeq \mathbf{Z} / 3 \mathbf{Z}$. But a 2-descent [S2], [Cr] shows that $E_{473}(\mathbf{Q})$ has rank 0. We deduce

$$
\amalg\left(E_{473} / \mathbf{Q}\right)[\phi] \simeq \mathbf{Z} / 3 \mathbf{Z} \quad \text { and } \quad \amalg\left(\widetilde{E}_{473} / \mathbf{Q}\right)[\widehat{\phi}] \simeq \mathbf{Z} / 3 \mathbf{Z} .
$$

Remark 4.4 According to the formulae and tables of Stephens [St], the above examples have $L\left(E_{A}, 1\right) \neq 0$. So the claims $\operatorname{rank} E_{A}(\mathbf{Q})=0$ could equally be deduced from the work of Coates-Wiles [CW].

Example 4.2 tells us that each of the curves

$$
\begin{aligned}
T[3,4,5]: & 3 x_{0}^{3}+4 x_{1}^{3}+5 x_{2}^{3}=0 \\
T[1,3,20]: & x_{0}^{3}+3 x_{1}^{3}+20 x_{2}^{3}=0 \\
T[1,4,15]: & x_{0}^{3}+4 x_{1}^{3}+15 x_{2}^{3}=0 \\
T[1,5,12]: & x_{0}^{3}+5 x_{1}^{3}+12 x_{2}^{3}=0
\end{aligned}
$$

is a counterexample to the Hasse Principle for smooth curves of genus 1 defined over Q. Selmer proves this without the need for a 2-descent. Instead he shows that the equations (10) are insoluble over $\mathbf{Q}$ by writing them as norm equations. As Cassels explains [CaI, §11] this is equivalent to performing a second descent, i.e. computing the middle group in

$$
\widetilde{E}_{A}(\mathbf{Q}) / \phi E_{A}(\mathbf{Q}) \subset \widehat{\phi} S^{(3)}\left(\widetilde{E}_{A} / \mathbf{Q}\right) \subset S^{(\phi)}\left(E_{A} / \mathbf{Q}\right)
$$

In fact Selmer's calculations suffice to show that $\amalg\left(E_{60} / \mathbf{Q}\right)(3) \simeq(\mathbf{Z} / 3 \mathbf{Z})^{2}$. In other words $\amalg\left(E_{60} / \mathbf{Q}\right)$ does not contain an element of order 9. More recent work of Rubin $[\mathrm{M}]$ improves this to $\amalg\left(E_{60} / \mathbf{Q}\right) \simeq(\mathbf{Z} / 3 \mathbf{Z})^{2}$.

Selmer also gave practical methods for computing the two right hand groups in

$$
E_{A}(\mathbf{Q}) / \widehat{\phi} \widetilde{E}_{A}(\mathbf{Q}) \subset \phi S^{(3)}\left(E_{A} / \mathbf{Q}\right) \subset S^{(\widehat{\phi})}\left(\widetilde{E}_{A} / \mathbf{Q}\right)
$$


Following Stephens [St] we write $g_{1}+1, \lambda_{1}^{\prime}+1, \lambda_{1}+1$ for the dimensions of the $\mathbf{Z} / 3 \mathbf{Z}$-vector spaces (11) and $g_{2}, \lambda_{2}^{\prime}, \lambda_{2}$ for the dimensions of the $\mathbf{Z} / 3 \mathbf{Z}$ vector spaces (12). Trivially we have $0 \leq g_{1} \leq \lambda_{1}^{\prime} \leq \lambda_{1}, 0 \leq g_{2} \leq \lambda_{2}^{\prime} \leq \lambda_{2}$ and rank $E_{A}(\mathbf{Q})=g_{1}+g_{2}$. Based on a large amount of numerical evidence, Selmer [S3] made the following

Conjecture 4.5 Let $A \geq 2$ be a cube-free integer. Let $E_{A}$ be the elliptic curve $x^{3}+y^{3}=A z^{3}$ defined over $\mathbf{Q}$. Then

Weak form. The second descent excludes an even number of generators, i.e. $\lambda_{1} \equiv \lambda_{1}^{\prime}(\bmod 2)$ and $\lambda_{2} \equiv \lambda_{2}^{\prime}(\bmod 2)$.

Strong form. The number of generators of infinite order for $E_{A}(\mathbf{Q})$ is an even number less than what is indicated by the first descent, i.e. $\lambda_{1}+\lambda_{2} \equiv$ $g_{1}+g_{2}(\bmod 2)$.

For $A=473$, Selmer found $\lambda_{1}=\lambda_{1}^{\prime}=\lambda_{2}=\lambda_{2}^{\prime}=1$ yet $g_{1}=g_{2}=0$. He was thus aware of the need to combine the contributions from $\phi$ and $\widehat{\phi}$ in the strong form of his conjecture.

Remark 4.6 In Heath-Brown's notation [HB] we have

$$
r(A)=\operatorname{rank} E_{A}(\mathbf{Q})=g_{1}+g_{2} \quad \text { and } \quad s(A)=\lambda_{1}+\lambda_{2} .
$$

By (9) the order of $S^{(\sqrt{-3})}\left(E_{A} / K\right)$ is $3^{s(A)+1}$ and in fact it is this relation that Heath-Brown uses to define $s(A)$. Naturally he writes the strong form of Selmer's conjecture as $r(A) \equiv s(A)(\bmod 2)$.

Now let $k$ be any number field. Conjecture 4.5 is equivalent to the case $k=\mathrm{Q}$ of the following

Conjecture 4.7 Let $A \in k^{*}$ not a perfect cube. Let $E_{A}$ be the elliptic curve $x^{3}+y^{3}=A z^{3}$ defined over $k$. Then

Weak form. The subgroup $\widehat{\phi}\left(\amalg\left(\widetilde{E}_{A} / k\right)[3]\right) \subset \amalg\left(E_{A} / k\right)[\phi]$ has index a perfect square. The same is true for $\phi\left(\amalg\left(E_{A} / k\right)[3]\right) \subset \amalg\left(\widetilde{E}_{A} / k\right)[\widehat{\phi}]$.

Strong form. The order of $\amalg\left(E_{A} / k\right)[\phi]$ multiplied by the order of $\amalg\left(\widetilde{E}_{A} / k\right)[\widehat{\phi}]$ is a perfect square.

In the next section we recall how Conjecture 4.7 follows from the work of Cassels, the strong form being conditional on the finiteness of $\amalg\left(E_{A} / k\right)$. 


\section{The Cassels-Tate pairing}

Let $E$ be an elliptic curve over a number field $k$. For $\phi: E \rightarrow E^{\prime}$ an isogeny of elliptic curves over $k$ we shall write $\widehat{\phi}: E^{\prime} \rightarrow E$ for the dual isogeny. Cassels $[\mathrm{CaIV}]$ defines an alternating bilinear pairing

$$
\langle,\rangle: \amalg(E / k) \times \amalg(E / k) \rightarrow \mathbf{Q} / \mathbf{Z}
$$

with the following non-degeneracy property.

Theorem 5.1 Let $\phi: E \rightarrow E^{\prime}$ be an isogeny of elliptic curves over $k$. Then $x \in \amalg(E / k)$ belongs to the image of $\widehat{\phi}: \amalg\left(E^{\prime} / k\right) \rightarrow \amalg(E / k)$ if and only if $\langle x, y\rangle=0$ for all $y \in \amalg(E / k)[\phi]$.

Proof. This was proved by Cassels $[\mathrm{CaIV}]$ in the case $\phi=[m]$ for $m$ a rational integer. The general case follows by his methods and is explained in $[\mathrm{F}]$.

The pairing was later generalised to abelian varieties by Tate, and so is known as the Cassels-Tate pairing. The most striking applications in the case of elliptic curves come from the following easy lemma.

Lemma 5.2 If a finite abelian group admits a non-degenerate alternating bilinear pairing, then its order must be a perfect square.

The weak form of Conjecture 4.7 is a special case of

Corollary 5.3 Let $\phi: E \rightarrow E^{\prime}$ be an m-isogeny of elliptic curves over $k$. Then the subgroup $\widehat{\phi}\left(\amalg\left(E^{\prime} / k\right)[m]\right) \subset \amalg(E / k)[\phi]$ has index a perfect square.

Proof. According to Theorem 5.1 the pairing (13) restricted to $\amalg(E / k)[\phi]$ has kernel $\widehat{\phi}\left(\amalg\left(E^{\prime} / k\right)[m]\right)$. We are done by Lemma 5.2 .

Let us assume that $\amalg(E / k)$ is finite. So by Theorem 5.1 and Lemma 5.2 the order of $\amalg(E / k)$ is a perfect square. If $\phi: E \rightarrow E^{\prime}$ is an isogeny of elliptic curves over $k$ then the same conclusions will hold for $E^{\prime}$. We define

$$
\langle,\rangle_{\phi}: \amalg(E / k) \times \amalg\left(E^{\prime} / k\right) \rightarrow \mathbf{Q} / \mathbf{Z} ; \quad(x, y) \mapsto\langle\phi x, y\rangle=\langle x, \widehat{\phi} y\rangle
$$

where the equality on the right is [CaVIII, Theorem 1.2]. The strong form of Conjecture 4.7 is a special case of 
Corollary 5.4 Let $\phi: E \rightarrow E^{\prime}$ be an isogeny of elliptic curves over $k$. If $\amalg(E / k)$ is finite then the order of $\amalg(E / k)[\phi]$ multiplied by the order of $\amalg\left(E^{\prime} / k\right)[\widehat{\phi}]$ is a perfect square.

Proof. According to Theorem 5.1 the left and right kernels of $\langle,\rangle_{\phi}$ are $\amalg(E / k)[\phi]$ and $\amalg\left(E^{\prime} / k\right)[\widehat{\phi}]$. We obtain a non-degenerate pairing

$$
\amalg(E / k) / \amalg(E / k)[\phi] \times \amalg\left(E^{\prime} / k\right) / \amalg\left(E^{\prime} / k\right)[\widehat{\phi}] \rightarrow \mathbf{Q} / \mathbf{Z} .
$$

We deduce that these quotients have the same order and are done since $\amalg(E / k)$ and $\amalg\left(E^{\prime} / k\right)$ each have order a perfect square.

Another well known consequence is

Corollary 5.5 Let $E$ be an elliptic curve over $k$ whose Tate-Shafarevich group is finite, and let $m$ be a rational integer. Then the order of $\amalg(E / k)[m]$ is a perfect square.

Proof. According to Theorem 5.1 the kernel of $\langle,\rangle_{m}$ is $\amalg(E / k)[m]$. We obtain a non-degenerate alternating pairing

$$
\amalg(E / k) / \amalg(E / k)[m] \times \amalg(E / k) / \amalg(E / k)[m] \rightarrow \mathbf{Q} / \mathbf{Z} .
$$

We apply Lemma 5.2 to this pairing and are done since $\amalg(E / k)$ has order a perfect square.

Remark 5.6 We could equally deduce Corollary 5.4 from Corollaries 5.3 and 5.5.

Proof of Theorem 1.1. Let $E$ be an elliptic curve over $k$ with complex multiplication by $\mathbf{Z}[\omega]$ and suppose that $[K: k]=2$. Lemma 2.1 tells us that

$$
\amalg(E / K)[\sqrt{-3}] \simeq \amalg(E / k)[\phi] \oplus \amalg(\widetilde{E} / k)[\widehat{\phi}] .
$$

Assuming $\amalg(E / k)$ is finite, Corollary 5.4 shows that the group on the right has order a perfect square. So the group on the left has order a perfect square, and this is precisely the statement of Theorem 1.1.

In the first of his celebrated series of papers, Cassels [CaI] defines a pairing $S^{(\sqrt{-3})}\left(E_{A} / K\right) \times S^{(\sqrt{-3})}\left(E_{A} / K\right) \rightarrow \mu_{3}$. It is of course a special case of the pairing (13). He uses it to prove the weak form of Conjecture 4.7 in the case $[K: k]=1$. However in the introduction to the same paper he misquotes the strong form of Selmer's conjecture. The statement he gives is equivalent to 
- If $[K: k]=1$ then the order of $\amalg\left(E_{A} / K\right)[\sqrt{-3}]$ is a perfect square.

It is this statement to which we have found a counterexample. It is possible that Cassels was mislead by earlier work of Selmer at a time when he did not appreciate the need to combine the contributions from $\phi$ and $\widehat{\phi}$ in the strong form of his conjecture.

Remark 5.7 It is tempting to try and prove Theorem 1.1 in the case $[K$ : $k]=1$ by imitating the proof of Corollary 5.5 . However the isogeny $[\sqrt{-3}]$ has dual $[-\sqrt{-3}]$ and this extra sign means that the pairing $\langle,\rangle_{\sqrt{-3}}$ is symmetric rather than alternating. Lemma 5.2 does not apply.

\section{A new example}

In this section we take $K=\mathbf{Q}(\omega)$. Let $E_{A}$ be the elliptic curve $x^{3}+y^{3}=A z^{3}$. We aim to find $A \in K$ such that the order of $\amalg\left(E_{A} / K\right)[\sqrt{-3}]$ is not a perfect square. As in Example 4.3 our method is to compare a 3-descent with a 2descent. The form of the curves $E_{A}$ makes the 3-descent easy. We use the results of $\S 3$ to compute the Selmer group $S^{(\sqrt{-3})}\left(E_{A} / K\right)$. For the 2-descent we would like to use John Cremona's program mwrank [Cr]. But mwrank is written specifically for elliptic curves over $\mathbf{Q}$, whereas Theorem 1.1 tells us that there are no examples of the required form with $A^{2} \in \mathbf{Q}$. Fortunately we were able to use a program of Denis Simon [Si1], [Si2], written using pari [BBBCO], that extends Cremona's work on 2-descents to general number fields (in practice of degrees 1 to 5 ).

We consider all cube-free $A \in \mathbf{Z}[\omega]$ with $A^{2} \notin \mathbf{Q}$ and $\operatorname{Norm}(A) \leq 150$. We ignore repeats of the form $\pm \sigma(A)$ for $\sigma \in \mathrm{Gal}(K / \mathbf{Q})$. In all 123 cases a calculation based on Lemmas 3.2 and 3.3 shows that $S^{(\sqrt{-3})}\left(E_{A} / K\right)$ is isomorphic to either $\mathbf{Z} / 3 \mathbf{Z}$ or $(\mathbf{Z} / 3 \mathbf{Z})^{2}$. In the 98 cases where $S^{(\sqrt{-3})}\left(E_{A} / K\right) \simeq \mathbf{Z} / 3 \mathbf{Z}$ it follows immediately that $\operatorname{rank} E_{A}(K)=0$. In the remaining 25 cases we run Simon's program. For 20 of these curves the program exhibits a point of infinite order. Since $E_{A}(K)$ has the structure of $\mathbf{Z}[\omega]$-module, we are able to deduce that rank $E_{A}(K)=2$. The remaining 5 cases are

$$
A= \pm(3+7 \omega), \pm(9+\omega), \pm(12+5 \omega), \pm(6+13 \omega), \pm(13+7 \omega)
$$

and their Galois conjugates. In each case Simon's program reports that $\operatorname{rank} E_{A}(K)=0$. Reducing modulo some small primes we find $E_{A}(K) \simeq \mathbf{Z} / 3 \mathbf{Z}$. 
Thus

$$
\amalg\left(E_{A} / K\right)[\sqrt{-3}] \simeq \mathbf{Z} / 3 \mathbf{Z} .
$$

For the remainder of this article we restrict attention to the first of these examples, namely $A=3+7 \omega$, and give further details of the descent calculations involved. In particular we establish the counterexample of the title in a way that is independent of Simon's program.

We begin by checking the above computation of $S^{(\sqrt{-3})}\left(E_{A} / K\right)$ for $A=$ $3+7 \omega$. Since $(A)$ is prime, Lemma 3.2 tells us that

$$
S^{(\sqrt{-3})}\left(E_{A} / K\right) \subset\langle\omega, 3+7 \omega\rangle .
$$

We check the local conditions at the primes $(\pi)$ and $(A)$ above 3 and 37 respectively.

- Since $37 \equiv 1(\bmod 9)$ we know that $\omega$ is a cube locally at $(A)$.

- Lemma 3.3 gives $\operatorname{im} \delta_{\pi}=\left\langle A, 1-\pi^{3}\right\rangle \subset K_{\pi}^{*} / K_{\pi}^{* 3}$. Since $A=\omega-\pi^{3}$ it is clear that $\omega$ belongs to this subgroup.

It follows that equality holds in (15) as required.

Given the provisional nature of Simon's program we have taken the liberty of writing out the 2-descent for $A=3+7 \omega$ in the style of Cassels [CaL, p.7273]. The curve $E_{A}$ has Weierstrass form

$$
Y^{2}=X^{3}-2^{4} 3^{3}(3+7 \omega)^{2} .
$$

The 2-descent takes place over the field $L=K(\delta)$ where $\delta^{3}=4(3+7 \omega)$. According to pari $[\mathrm{BBBCO}]^{1}, L$ has class number $h=3$, and fundamental units

$$
\begin{aligned}
& \eta_{1}=(-7-3 \omega)+(-3-2 \omega) \delta+(-2+\omega) \delta^{2} / 2 \\
& \eta_{2}=(-7-3 \omega)+(2-\omega) \delta+(3+2 \omega) \delta^{2} / 2 .
\end{aligned}
$$

Furthermore pari is able to certify these results, independent of any conjecture. We have chosen $\eta_{1}$ and $\eta_{2}$ to be $K$-conjugates. They have minimal polynomial

$$
x^{3}+(21+9 \omega) x^{2}+(102-165 \omega) x-1 .
$$

If $(X, Y)=\left(r / t^{2}, s / t^{3}\right)$ is a solution of (16), with fractions in lowest terms, then a common prime divisor of any two of

$$
r-3 \delta^{2} t^{2}, \quad r-3 \omega \delta^{2} t^{2}, \quad r-3 \omega^{2} \delta^{2} t^{2}
$$

\footnotetext{
${ }^{1}$ These calculations were performed using Version 2.0.20 (beta)
} 
must divide $2(1-\omega)(3+7 \omega)$. Since $2,(1-\omega),(3+7 \omega)$ ramify completely, $r-3 \delta^{2} t^{2}$ must be a perfect ideal square. Since $h$ is odd it follows that $S^{(2)}(E / K)$ is a subgroup of $\left\langle-1, \eta_{1}, \eta_{2}\right\rangle \subset L^{*} / L^{* 2}$. We claim that $S^{(2)}(E / K)$ is trivial. By considering norms from $L$ to $K$, it suffices to show that the equation

$$
r-3 \delta^{2} t^{2}=\eta \alpha^{2} \quad \text { with } \eta=\eta_{1}, \eta_{2} \text { or } 1 /\left(\eta_{1} \eta_{2}\right)
$$

is insoluble for $r, t \in K$ and $\alpha \in L$. The action of $\operatorname{Gal}(L / K)$ shows that we need only consider the case $\eta=\eta_{1}$. Put $\alpha=u+v \delta+w \delta^{2}$. Equating coefficients of powers of $\delta$ we obtain

$$
\begin{aligned}
& 0=(-3-2 \omega) u^{2}+(-14-6 \omega) u v+(-26-36 \omega) v^{2} \\
& +(-52-72 \omega) u w+(40-104 \omega) v w+(-148 \omega) w^{2} \\
& -3 t^{2}=((-2+\omega) / 2) u^{2}+(-6-4 \omega) u v+(-7-3 \omega) v^{2} \\
& +(-14-6 \omega) u w+(-52-72 \omega) v w+(20-52 \omega) w^{2} .
\end{aligned}
$$

On putting

$$
\begin{aligned}
u & =(-8+6 \omega) e+(-6-34 \omega) f+(-20+15 \omega) g \\
v & =(-4-4 \omega) e+(12+4 \omega) f+(-10-11 \omega) g \\
w & =(1-\omega) e+(1+4 \omega) f+(2-2 \omega) g
\end{aligned}
$$

in the first equation, it becomes

$$
0=(3+7 \omega) g^{2}-16 e f .
$$

Hence there are $m, n$ such that

$$
e: f: g=m^{2}:(3+7 \omega) n^{2}: 4 m n \text {. }
$$

On substituting into the second equation, we get

$$
\begin{aligned}
-3 t^{2}=2(-1-4 \omega) m^{4}+8 & -4+3 \omega) m^{3} n+4(21+12 \omega) m^{2} n^{2} \\
& +8(4-3 \omega) m n^{3}+2(-33-40 \omega) n^{4} .
\end{aligned}
$$

But this is impossible in $K_{2}$. Hence $S^{(2)}\left(E_{A} / K\right)$ is trivial and rank $E_{A}(K)=$ 0 as claimed.

\section{Acknowledgements}

The author would like to thank Laura Basile, Alexei Skorobogatov and Sir Peter Swinnerton-Dyer for useful conversations. 


\section{References}

[BF] C.L. Basile and T.A. Fisher, Diagonal cubic equations in four variables with prime coefficients, Rational points on algebraic varieties, 112, Progr. Math., 199, Birkhäuser, Basel, 2001.

[BBBCO] C. Batut, K. Belabas, D. Bernardi, H. Cohen and M. Olivier, pari/gp, a computer algebra package, http://www.parigp-home.de

[CaI] J.W.S. Cassels, Arithmetic on curves of genus 1, I. On a conjecture of Selmer, J. Reine Angew. Math. 202 (1959), 52-99.

[CaIV] J.W.S. Cassels, Arithmetic on curves of genus 1, IV. Proof of the Hauptvermutung, J. Reine Angew. Math. 211 (1962), 95-112.

[CaVIII] J.W S. Cassels, Arithmetic on curves of genus 1, VIII. On conjectures of Birch and Swinnerton-Dyer, J. Reine Angew. Math. 217 (1965), 180-199.

[CaL] J.W.S. Cassels, Lectures on elliptic curves, LMSST 24, Cambridge University Press, Cambridge, 1991.

[CF] J.W.S. Cassels and A. Fröhlich (Eds.), Algebraic number theory, Academic Press, London, 1967.

[CW] J. Coates and A. Wiles, On the conjecture of Birch and SwinnertonDyer. Invent. Math. 39 (1977), no. 3, 223-251.

[Cr] J.E. Cremona, mwrank, a program for performing 2-descent on elliptic curves over $\mathbf{Q}$,

http://www.maths.nottingham.ac.uk/personal/jec/ftp/progs

[F] T.A. Fisher, The Cassels-Tate pairing and the Platonic solids, to appear in J. Number Theory.

[HB] D.R. Heath-Brown, The solubility of diagonal cubic Diophantine equations, Proc. London Math. Soc. (3) 79 (1999), no. 2, 241-259.

[K] N. Koblitz, Introduction to elliptic curves and modular forms, GTM 97. Springer-Verlag, New York, 1993. 
[M] B. Mazur, On the passage from local to global in number theory. Bull. Amer. Math. Soc. 29 (1993), no. 1, 14-50.

[N] J. Nekovář Class numbers of quadratic fields and Shimura's correspondence, Math. Ann. 287 (1990), no. 4, 577-594.

[S1] E.S. Selmer, The diophantine equation $a x^{3}+b y^{3}+c z^{3}=0$, Acta Math. 85 (1951), 203-362.

[S2] E.S. Selmer, The diophantine equation $a x^{3}+b y^{3}+c z^{3}=0$, completion of the tables, Acta Math. 92 (1954), 191-197.

[S3] E.S. Selmer, A conjecture concerning rational points on cubic curves, Math. Scand. 2 (1954), 49-54.

[Sil] J.H. Silverman, The arithmetic of elliptic curves, GTM 106, SpringerVerlag, New York, 1986.

[Si1] D. Simon, Computing the rank of elliptic curves over number fields, LMS J. Comput. Math. 5 (2002), 7-17.

[Si2] D. Simon, ell.gp, a program for calculating the rank of elliptic curves over number fields, http://www.math.unicaen.fr/ simon

[St] N.M. Stephens, The diophantine equation $X^{3}+Y^{3}=D Z^{3}$ and the conjectures of Birch and Swinnerton-Dyer, J. Reine Angew. Math. 231 (1968), 121-162.

[SwD] H.P.F. Swinnerton-Dyer, The solubility of diagonal cubic surfaces, Ann. Sci. École Norm. Sup. (4) 34 (2001), no. 6, 891-912. 